\title{
The acaricidal speed of kill of orally administered fluralaner against poultry red mites (Dermanyssus gallinae) on laying hens and its impact on mite reproduction
}

\author{
Maria D. Brauneis, Hartmut Zoller, Heike Williams,' Eva Zschiesche and Anja R. Heckeroth
}

\begin{abstract}
Background: Dermanyssus gallinae, the poultry red mite, is a growing threat to chickens in poultry farms. This nocturnal hematophagous ectoparasite has a rapid rate of proliferation with a negative impact on the birds' health, welfare and productivity resulting in severe economic consequences for poultry farmers. A study was performed with fluralaner, a novel systemic ectoparasiticide, to evaluate its effect on mite vitality and reproduction after oral administration to laying hens.

Methods: Sixteen healthy hens were randomly allocated to two study groups $(n=8)$. One group was orally treated with fluralaner by gavage at a dose of $0.5 \mathrm{mg} / \mathrm{kg}$ bodyweight twice 7 days apart. The negative control group received no treatment. Hens in each group were repeatedly infested with approximately 200 unfed adult $D$. gallinae at $1,5,8,12,15,19,22$ and 26 days after the initial administration. After infestation and feeding for $2.5 \mathrm{~h}, 25$ engorged mites per hen were collected and incubated in tubes. Mites were assessed for vitality (dead/live) at 4, 8, 12, and $24 \mathrm{~h}$ after each infestation. Tubes containing eggs and/or living mites were incubated another 8 days for assessment of mite reproductive capacity.

Results: Fluralaner demonstrated a fast speed of kill in mites within $4 \mathrm{~h}$ post-infestation for 12 days after treatment initiation. An efficacy (mite mortality) of $98.7-100 \%$ was achieved. At 15 days after treatment initiation, 100\% efficacy was achieved within $24 \mathrm{~h}$ post-infestation, and no mite oviposition occurred during this period. Nineteen days after treatment initiation, the mites' ability to generate nymphs was reduced by $90.8 \%$, which decreased to $<24.1 \%$ at later infestations.

Conclusions: Fluralaner administered orally to hens twice, 7 days apart, provides efficacy against experimental poultry red mite infestation for at least 2 weeks. The demonstrated rapid speed of kill results in substantial depletion of the mites' oviposition and suggests that fluralaner can be an effective tool in the control of $D$. gallinae, one of the most urgent problems in poultry farms.
\end{abstract}

Keywords: Poultry red mite, Dermanyssus gallinae, Laying hen, Fluralaner, Drinking water, Speed of kill, Reproduction

* Correspondence: heike.williams@msd.de

MSD Animal Health Innovation GmbH, Research Antiparasitics, Zur Propstei,

55270 Schwabenheim, Germany International License (http://creativecommons.org/licenses/by/4.0/), which permits unrestricted use, distribution, and reproduction in any medium, provided you give appropriate credit to the original author(s) and the source, provide a link to the Creative Commons license, and indicate if changes were made. The Creative Commons Public Domain Dedication waiver (http://creativecommons.org/publicdomain/zero/1.0/) applies to the data made available in this article, unless otherwise stated. 


\section{Background}

Dermanyssus gallinae (De Geer, 1778), the poultry red mite, is a growing threat to chickens in poultry farms. This blood-sucking ectoparasite especially affects hens in the egg-laying industry, but also hobby flocks $[1,2]$. Estimates of poultry red mite prevalence suggest that up to $94 \%$ of commercial housing systems are infested [3]. Dermanyssus gallinae harms poultry either directly through blood-feeding or indirectly as a potential vector for numerous pathogens [4-6]. The poor health condition of mite infested poultry causes major economic losses for the egg industry (estimated around $€ 230$ million per year for Europe), due to diminished egg production and poor egg quality [7]. Moreover, mite-infested poultry houses can negatively impact farm workers' health by exposing them to the parasite's bite, an increasing risk for a zoonotic disease [8,9]. In severe cases workers suffer amongst others from persistent skin irritation and itching dermatitis $[1,2,10]$.

Dermanyssus gallinae is a ubiquitous hematophagous ectoparasite, preferably seeking its hosts at night, with almost all mite life stages, except for larvae, feeding on the blood of hens [1, 11]. After nocturnal feeding on hens for only 0.5 to $1.5 \mathrm{~h}$ mites retreat into cracks and crevices of nest boxes or the wider surroundings of the poultry house to digest their blood meal, mate and reproduce [12-14]. Female mites can feed and reproduce repeatedly, laying up to 30 eggs during their life time; the mite's rapid life-cycle further contributes to the status of $D$. gallinae as a pest $[1,10]$. Under optimal conditions, mite development from egg to adult takes around 7 days and includes 5 stages, i.e. eggs, larvae, protonymphs, deutonymphs and adults $[1,14]$. Thus, a weekly doubling of the mite population is possible, and high parasite burdens can be reached ( $\geq 150,000$ mites per bird are reported) $[10,12]$. The impact of mite infestation on the birds' health and well-being are serious: anemia through substantial blood loss, restlessness, feather picking, stress-caused aggression, weight loss and even death can occur. The production of blood-stained eggs and the reduction in egg size and quality are additional negative consequences for the farmers $[1,4,13]$.

Because poultry red mites are resistant to desiccation they are able to survive without feeding in the absence of a host for up to 8 months [1]. Consequently, remaining mites can continuously multiply and build up a population over many production cycles, which often makes a one-time treatment of the poultry houses against D. gallinae challenging. Therefore, a fast, thorough and lasting elimination of the entire mite population is essential to effectively control this poultry pest. An acaricide with a fast mite killing effect which also interrupts the mites' reproduction could achieve sustainable $D$. gallinae control in poultry farms.
Fluralaner, a member of the isoxazoline class, is a potent inhibitor of ligand-gated chloride channels with a high selectivity for the nervous system of acarids and insects [15-18]. Marketed for both, dogs and cats, fluralaner acts systemically against ticks and fleas [19]. Fluralaner also demonstrates the ability to considerably impact the reproduction of fleas, e.g. after oral administration to dogs $[16,17,20,21]$.

For poultry, a systemic medication via drinking water is a common and convenient treatment procedure and also a safe and simple method for $D$. gallinae control $[22,23]$. This study was conducted to investigate fluralaner's efficacy against poultry red mites including its speed of kill and possible effects on mite reproduction after oral administration to hens.

\section{Methods}

\section{Study design}

This study investigated the acaricidal efficacy of fluralaner against the poultry red mite, $D$. gallinae, including its speed of kill and impact on mite reproduction after oral administration to hens.

Sixteen laying hens were obtained from a commercial provider (Lohmann Selected Leghorn). During an acclimatization period they were randomly assigned to 2 study groups of 8 hens each (1 treatment group and 1 control group) based on their health status and body weight (range 960-1580 g). The hens were between 20 and 21 weeks old, and had not been exposed to any ectoparasite control product before start of the study. The hens were initially housed within their study group in pens enriched with nests, wooden shavings as nesting material, perches and cardboard. Due to a continuing social incompatibility among the hens, they were singlehoused from day 13 after initial dose administration to the end of the animal phase. The housing room of hens was equipped with an artificial red light for $12 \mathrm{~h}$ per day and ambient temperature ventilation system.

All hens were clinically examined and weighed 1 day before the initial fluralaner dose administration (day 0) and at the end of the animal phase. Prior to the second administration (day 7), hens in the fluralaner group were weighed again for dose calculation purposes. The hens' general health was monitored daily during the study.

\section{Treatment}

The hens in the treatment group were administered fluralaner twice, and administrations were performed 7 days apart. Prior to both administrations, a stock solution containing $10 \mathrm{mg}$ fluralaner/ml was diluted with tap water to obtain the intended dosing concentration of $0.2 \mathrm{mg}$ fluralaner $/ \mathrm{ml}$. On day 0 and day 7 , hens of the treatment group were orally administered at a dose of $0.5 \mathrm{mg}$ fluralaner $/ \mathrm{kg}$ body weight by gavage, and hens of 
the negative control group remained untreated. The administration volume per hen was calculated based on each hens' body weight. Approximately 1, 6 and $24 \mathrm{~h}$ after the oral administration, hens were observed for possible adverse reactions, e.g. changes in general health condition and behavior.

\section{Mite infestation, collection and incubation}

The poultry red mite (D. gallinae) isolate originated from a commercial layer farm in Germany and has been maintained as a colony at MSD Animal Health since 2001. The mite isolate is known to be susceptible to organophosphates, carbamates and spinosyns, but less susceptible to synthetic pyrethroids. All mites used for infestation showed natural mobility and were starved for 3 days prior to infestation. Six out of 8 hens in each study group were infested with approximately 200 unfed mites per infestation event on days 1, 5, 8, 12, 15, 19, 22 and 26 after the initial fluralaner administration. For an infestation period of $2.5 \mathrm{~h}$, the hens were kept in separate polystyrene boxes $(57 \times 44 \times 35 \mathrm{~cm}$ in size $)$ and in the dark to enable mite feeding. The bottom of the box was outlined with a metal grid to prevent mites being damaged by the hen, and a mite-proof, but airpermeable lid ensured sufficient air flow during the infestation process.

At the end of an infestation period, hens were removed from the boxes and random aliquots of $25( \pm 2)$ visibly engorged, adult mites were collected from each hens' box by aspiration using a vacuum pump. Each mite aliquot was divided to 5 glass tubes of approximately 5 mites each. The glass tubes contained a colored paper strip to improve the visibility of mites in the tube thereby facilitating the monitoring of adult mites and their emerging juvenile stages. Mites in glass tubes were incubated in a climate chamber at $28{ }^{\circ} \mathrm{C}$ and $85 \%$ relative humidity, and were only temporarily removed from the chamber for the assessments of mite vitality and reproduction parameters. The incubation of mites was continued for 8 days to enable an emergence of nymphs.

\section{Assessment of adult mite vitality}

Assessment of mite vitality was conducted repeatedly, i.e. at $4,8,12$ and $24 \mathrm{~h}$ after a hen's infestation had started, by counting the number of live/dead adult mites in a glass tube using a binocular (magnification 8- to 40fold). The mites were classified as dead if they were in a dorsal position or no motility was detected.

\section{Assessment of mite reproduction}

In conjunction with the mite vitality assessment conducted at $24 \mathrm{~h}$ post-infestation (see above), additional parasite counts were performed, including the numbers of live females/males, dead females/males, and viable eggs.
Thereafter, daily counts of further developmental life stages were conducted during the incubation period, i.e. 8 days after an infestation, including the numbers of live females/males, dead females/males, viable eggs, live/dead larvae, and live/dead protonymphs.

\section{Data analysis}

The descriptive data analysis was performed by means of Microsoft Excel $^{\circ} 2010$ (Microsoft Corporation, Redmond, WA, USA) and statistical tests were performed using the software package $\mathrm{SAS}^{\circ}$ (SAS Institute Inc., Cary, NC, USA, release 9.4). The treatment unit was the individual animal. The statistical unit was the individual mite for calculations of efficacy (mite mortality) and reproduction parameters.

\section{Calculation of acaricidal efficacy}

The acaricidal efficacy was calculated as mite mortality $(\mathrm{MM})$ at $4,8,12$, and $24 \mathrm{~h}$ after each infestation, i.e. on days $1,5,8,12,15,19,22$ and 26 post-administration. The following formula was used:

$$
\operatorname{MM}(\%)=\left(\mathrm{MR}_{\mathrm{T}}-\mathrm{MR}_{\mathrm{C}}\right) /\left(1-\mathrm{MR}_{\mathrm{C}}\right) \times 100
$$

where $M R$ is the adult mite mortality rate in the treatment group $\left(M R_{T}\right)$ and the control group $\left(M R_{C}\right)$.

MR was calculated for both study groups as follows:

$$
\mathrm{MR}(\%)=\mathrm{DM} / \mathrm{CM} \times 100
$$

where DM is the number of dead mites per group, and $\mathrm{CM}$ is the number of collected mites per group.

To confirm the validity of the efficacy results, adult mite mortality rates in both study groups were compared at each time point using Fisher's exact test, one-sided with the level of significance set to $\alpha=0.025$.

\section{Calculation of effects on reproduction parameters}

Treatment effects on the reproductive capacity of $D$. gallinae were calculated by quantitative comparison of the emerging juvenile stages (eggs, larvae, protonymphs) in the treatment to the control group. The reproduction parameters were calculated as (i) reduction of eggs per live female mite at $24 \mathrm{~h}$ post-infestation and at 8 days post-infestation; (ii) reduction of larval hatchability; (iii) reduction of nymphal conversion; and (iv) reduction of nymphal emergence. These calculations were only performed for infestations on days 19, 22 and 26 postadministration because no live mites or eggs were found in the treated group at earlier infestation events, i.e. day 1 to day 15 post-administration. 
Reduction of eggs per live females (RELF)

RELF was calculated (a) $24 \mathrm{~h}$ post-infestation and (b) 8 days post-infestation as follows:

$$
\operatorname{RELF}(\%)=\left(\operatorname{ELF}_{\mathrm{C}}-\mathrm{ELF}_{\mathrm{T}}\right) / \mathrm{ELF}_{\mathrm{T}} \times 100
$$

where ELF is the number of eggs per live female mite, in the treatment group $\left(\mathrm{ELF}_{\mathrm{T}}\right)$ and the control group $\left(E^{2} F_{C}\right)$.

For (b), ELF was calculated daily ${ }_{\mathrm{d}}$ ) during the 8-day incubation period as the sum of newly observed eggs $\left(\mathrm{EN}_{\mathrm{d}}\right.$, calculation see below) divided by the sum of live females $\left(\mathrm{LFM}_{\mathrm{d}}\right)$ :

$$
E L F=\frac{\sum_{d=1}^{8} E_{d}}{\sum_{d=1}^{8} L_{d}}
$$

\section{Reduction of larval hatchability (RLH)}

RLH is an indicator for the egg viability and was calculated as follows:

$$
\operatorname{RLH}(\%)=\left(\mathrm{LH}_{\mathrm{C}}-\mathrm{LH}_{\mathrm{T}}\right) / \mathrm{LH}_{\mathrm{T}} \times 100
$$

where LH is the larval hatch, in the treatment group $\left(\mathrm{LH}_{\mathrm{T}}\right)$ and in the control group $\left(\mathrm{LH}_{\mathrm{C}}\right)$.

LH contributes to subsequent molting of larvae and was calculated daily $(\mathrm{d}$ ) during the 8-day incubation period as the sum of newly molted larvae $\left(\mathrm{LH}_{\mathrm{d}}\right)$ divided by the sum of newly observed eggs $\mathrm{EN}_{\mathrm{d}}$ :

$$
\% \mathrm{LH}=\frac{\sum_{\mathrm{d}=2}^{8} \mathrm{LH}_{\mathrm{d}}}{\sum_{\mathrm{d}=1}^{8} \mathrm{EN}_{\mathrm{d}}} \times 100
$$

$\mathrm{EN}_{\mathrm{d}}$ was calculated as follows:

$$
\mathrm{EN}_{\mathrm{d}}=\sum_{\mathrm{T}=1}^{30}\left\{\begin{array}{cl}
\mathrm{E}_{\mathrm{Td}} & \text { if } \mathrm{d}=1(\text { on d } 1) \\
\mathrm{E}_{\mathrm{Td}}+\mathrm{LH}_{\mathrm{Td}}-\mathrm{E}_{\mathrm{T}(\mathrm{d}-1)} & \text { if } \mathrm{d}>1 \wedge \mathrm{E}_{\mathrm{Td}}+\mathrm{LH}_{\mathrm{Td}} \geq \mathrm{E}_{\mathrm{T}(\mathrm{d}-1)} \\
0 & \text { else }
\end{array}\right.
$$

where $E_{T d}$ is the number of eggs, and $\mathrm{LH}_{\mathrm{Td}}$ is the number of newly molted larvae counted per tube $\left(_{\mathrm{T}}\right)$ and day $\left({ }_{\mathrm{d}}\right)$.

$\mathrm{LH}_{\mathrm{d}}$ was calculated as follows: where $\mathrm{L}_{\mathrm{Td}}$ is the number of larvae, and $\mathrm{N}_{\mathrm{Td}}$ the number of nymphs counted per tube $\left({ }_{\mathrm{T}}\right)$ and day $\left({ }_{\mathrm{d}}\right)$. The first day of incubation was excluded from the $\mathrm{LH}_{\mathrm{d}}$ calculation $(\mathrm{d}-1$, see formula above) because at this point in time a larval hatch from eggs is unlikely to occur.

\section{Reduction of nymphal conversion (RNC)}

$\mathrm{RNC}$ is an indicator for the reduction of larvae molting into nymphs and was calculated as follows:

$$
\operatorname{RNC}(\%)=\left(\mathrm{NCR}_{\mathrm{C}}-\mathrm{NCR}_{\mathrm{T}}\right) / \mathrm{NCR}_{\mathrm{T}} \times 100
$$

where NCR is the nymphal conversion ratio, in the treatment group $\left(\mathrm{NCR}_{\mathrm{T}}\right)$ and the control group $\left(\mathrm{NCR}_{\mathrm{C}}\right)$.

$\mathrm{NCR}$ is an indicator for the magnitude of larvae developing into protonymphs and was calculated as follows:

$$
\mathrm{NCR}=\frac{\sum_{\mathrm{d}=1}^{8} \sum_{\mathrm{T}=1}^{30} \mathrm{~N}_{\mathrm{Td}}}{\sum_{\mathrm{d}=1}^{8} \mathrm{LH}_{\mathrm{d}}} \times 100
$$

where $\mathrm{N}_{\mathrm{Td}}$ is the number of nymphs per tube $\left(_{\mathrm{T}}\right)$ and day $\left({ }_{d}\right)$; (see above for $\mathrm{LH}_{\mathrm{d}}$ calculation).

\section{Reduction of nymphal emergence (RNE)}

RNE includes all other reproduction parameters (RELF, RLH, and RNC) and was calculated at the end of the 8day incubation period as follows:

$$
\operatorname{RNE}(\%)=\left(\mathrm{NpF}_{\mathrm{C}}-\mathrm{NpF}_{\mathrm{T}}\right) / \mathrm{NpF}_{\mathrm{C}} \times 100
$$

where $\mathrm{NpF}$ is the number of nymphs per female, in the treatment group $\left(\mathrm{NpF}_{\mathrm{T}}\right)$ and the control group $\left(\mathrm{NpF}_{\mathrm{C}}\right)$.

$\mathrm{NpF}$ was calculated as follows:

$\mathrm{NpF}=\mathrm{NE} / \mathrm{FMI}$, where NE is the total number of nymphs emerged, and FMI is the total number of female mites incubated at each infestation.

\section{Results}

Acaricidal efficacy and speed of kill

Figure 1 shows both, acaricidal efficacy against adult mites and speed of kill. For 12 days after the fluralaner administrations, efficacy was $98.7-100 \%$ at all assessment times, i.e. conducted at $4,8,12$ and 24 h postinfestation. At 15 days, $82.6 \%$ efficacy was achieved at $4 \mathrm{~h}$ post-infestation that increased to $95.2 \%$ ( $8 \mathrm{~h}$ ), $99.3 \%$ $(12 \mathrm{~h})$ and $100 \%$ at $24 \mathrm{~h}$ post-infestation. At 19 days

$$
\mathrm{LH}_{\mathrm{d}}=\sum_{\mathrm{T}=1}^{30}\left\{\begin{array}{cl}
\left(\mathrm{L}_{\mathrm{Td}}-\mathrm{L}_{\mathrm{T}(\mathrm{d}-1)}\right)+\left(\mathrm{N}_{\mathrm{Td}}-\mathrm{N}_{\mathrm{T}(\mathrm{d}-1)}\right) & \text { if } \mathrm{N}_{\mathrm{Td}}-\mathrm{N}_{\mathrm{T}(\mathrm{d}-1)} \geq 0 \wedge \mathrm{N}_{\mathrm{Td}}-\mathrm{N}_{\mathrm{T}(\mathrm{d}-1)} \geq \mathrm{L}_{\mathrm{T}(\mathrm{d}-1)}-\mathrm{L}_{\mathrm{Td}} \\
\mathrm{L}_{\mathrm{Td}}-\mathrm{L}_{\mathrm{T}(\mathrm{d}-1)} & \text { if } \mathrm{N}_{\mathrm{Td}}-\mathrm{N}_{\mathrm{T}(\mathrm{d}-1)}<0 \wedge \mathrm{L}_{\mathrm{Td}}-\mathrm{L}_{\mathrm{T}(\mathrm{d}-1)} \geq 0 \\
0 & \text { else }
\end{array}\right.
$$




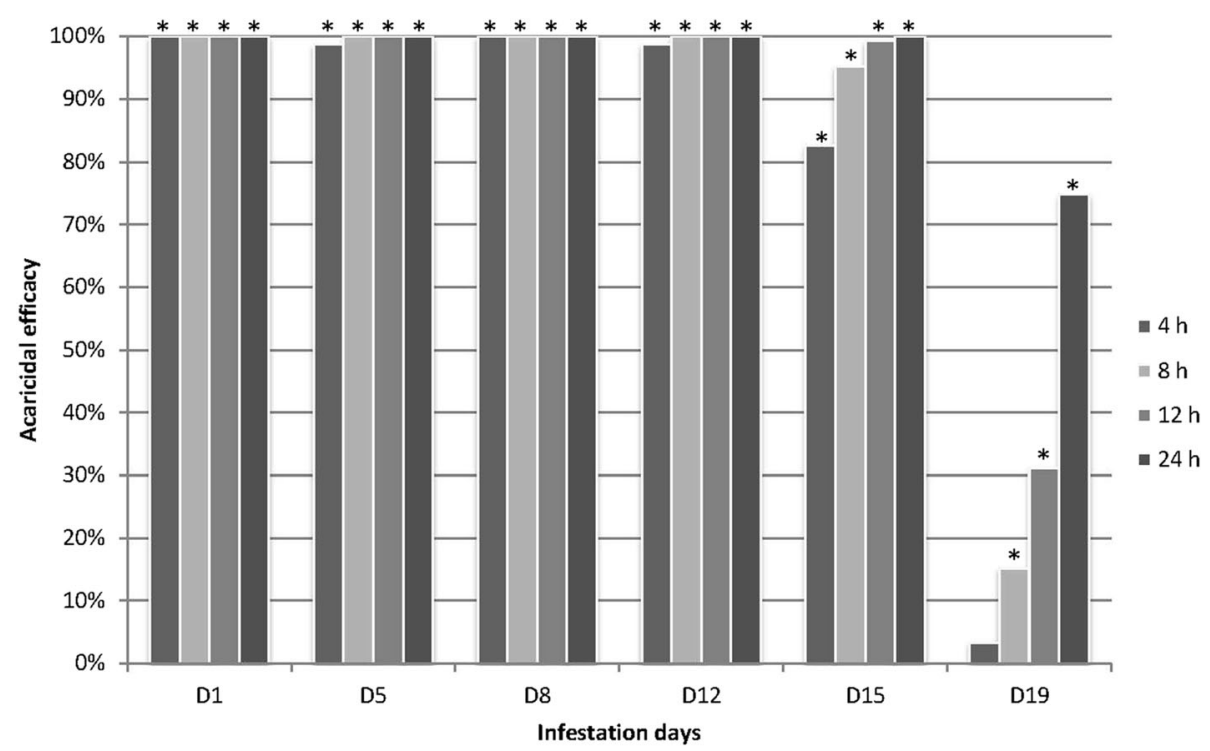

Fig. 1 Acaricidal efficacy (\%) after feeding of mites on fluralaner-treated hens. Assessment time points of the mites' vitality: 4, 8, 12 and $24 \mathrm{~h}$. $P<0.0001$

efficacy was $3.3 \%(4 \mathrm{~h}), 15.2 \%(8 \mathrm{~h}), 31.1 \%(12 \mathrm{~h})$ and $74.8 \%(24 \mathrm{~h})$. Further acaricidal efficacy assessments were omitted, i.e. on days 22 and 26 , because efficacies $<90 \%$ were observed for the infestation event on day 19 (Fig. 1).

A significant difference between study groups with $P<$ 0.0001 was shown for each time point except for mite mortality at $4 \mathrm{~h}$ after the infestation on day 19 (efficacy $3.3 \%, P=0.0292$, Fisher's exact test, one-sided).

\section{Mite reproduction parameters}

For 15 days after fluralaner administrations, $100 \%$ of mites died within $24 \mathrm{~h}$ following the infestation and no oviposition occurred in the fluralaner-treated group. Thus, the reproduction parameters RELF, RLH, RNC, and RNE were derived from later infestations conducted on days 19, 22 and 26.

Reduction of eggs per live female mite (RELF) assessed at $24 \mathrm{~h}$ post-infestation was $82.2 \%$ (day 19), 3.6\% (day 22) and $35.4 \%$ (day 26). RELF assessed 8 days postinfestation was $48.1 \%$ (day 19) and thereafter $\leq 15.9 \%$ (Table 1). Reduction of larval hatchability (RLH) was $\leq 2.6 \%$ (Table 2). Reduction of nymphal conversion

Table 1 Reduction of eggs per live female after mite feeding on treated hens. Assessment 8 days post-infestation

\begin{tabular}{llll}
\hline Infestation day & ELFC $_{C}$ & ELF $_{T}$ & RELF (\%) \\
\hline D19 & 0.8 & 0.4 & 48.1 \\
D22 & 0.8 & 0.7 & 15.9 \\
D26 & 0.8 & 0.7 & 10.6 \\
\hline
\end{tabular}

Abbreviations: ELF, eggs per live female; ${ }_{\mathrm{C}}$ control group; ${ }_{\mathrm{T}}$, treatment group; RELF, reduction of eggs per live female mite
(RNC) was $\leq 4.6 \%$ (Table 3). Reduction of nymphal emergence (RNE) was $90.8 \%$ (day 19) and thereafter $\leq 24.2 \%$ (Table 4 ).

Figure 2 illustrates the composition of the mite populations in the treated group in comparison to that of the control group following the infestations on day 19, 22 and 26 during the 8-day incubation period. After the infestation on day 19, the mite population developing in the treated group was considerably smaller than in the control group. In both study groups the highest egg number was found on incubation day $\mathrm{d} 3$, the highest number of larvae on incubation day $\mathrm{d} 4$, and the highest number of nymphs emerged on incubation day d8 (Fig. 2).

No adverse reactions were observed in any of the study animals that were considered to be treatment related during the post-treatment observation period.

\section{Discussion}

The preferred method for administering medical treatment in the poultry industry is orally via drinking water. So far this administration method has not been used for veterinary medicinal products in the treatment of the

Table 2 Reduction of larval hatchability after mite feeding on treated hens

\begin{tabular}{llll}
\hline Infestation day & $\mathrm{LH}_{\mathrm{C}}(\%)$ & $\mathrm{LH}_{\mathrm{T}}(\%)$ & $\mathrm{RLH}(\%)$ \\
\hline D19 & 94.5 & 93.6 & 0.9 \\
D22 & 91.6 & 90.5 & 1.2 \\
D26 & 93.9 & 91.4 & 2.6 \\
\hline
\end{tabular}

Abbreviations: $\mathrm{LH}$, larval hatch; ${ }_{\mathrm{C}}$ control group; ${ }_{\mathrm{T}}$, treatment group; $\mathrm{RLH}$, reduction of larval hatchability 
Table 3 Reduction of nymphal conversion after mite feeding on treated hens

\begin{tabular}{llll}
\hline Infestation day & $\mathrm{NCR}_{C}(\%)$ & $\mathrm{NCR}_{\mathrm{T}}(\%)$ & $\mathrm{RNC}(\%)$ \\
\hline D19 & 96.5 & 92.0 & 4.6 \\
D22 & 92.4 & 92.0 & 0.4 \\
D26 & 95.4 & 95.0 & 0.3
\end{tabular}

Abbreviations: NCR, nymphal conversion ratio; $\mathrm{c}$ control group; ${ }_{\mathrm{T}}$, treatment group; $\mathrm{RNC}$, reduction of nymphal conversion

poultry red mite, D. gallinae. The current standard approach to control $D$. gallinae in poultry houses is a topically applied premise treatment by spraying of a pesticide, such as phoxim or spinosad, or dusting with products based on silicon dioxide; the latter can serve as an alternative to the use of targeted chemical acaricides [24-26]. Topical premise treatments have limitations regarding the extent of their antiparasitic effects, because the parasites' hide-outs can be inaccessible with a spray or dust. As a result, some mites remain untreated and serve as a reservoir that is able to quickly build up a new bird-harming population in the stable [12, 27]. In this case, the labor-intense practice of topical premise treatment has to be performed more frequently [26]. Some premise treatments are approved to be performed in the presence of animals, and this can cause an additional disturbance of the birds, interrupting their normal behavior, and may temporarily impact food intake and productivity $[24,26]$. Resistance development in poultry red mite to acaricides, such as organophosphates, can also play a role in the failure of mite treatment in poultry houses [13].

A systemic treatment approach that is able to prevent mite reproduction could help to overcome the current limitations in D. gallinae control, because any vital blood-feeding mite stage in a poultry house will inevitably feed on a treated bird, and become exposed to the systemic acaricide. Furthermore, an acaricidal effect that acts quickly enough would disrupt the mite's egg production. The resulting beneficial effects are improved poultry health and well-being, along with a reduced potential of personnel being exposed to mite bites while working on poultry farms.

Oral administration of fluralaner is routinely used in dogs to provide a safe and fast acting antiparasitic

Table 4 Reduction of nymph emergence after mite feeding on treated hens

\begin{tabular}{llll}
\hline Infestation day & $\mathrm{NpF}_{\mathrm{C}}$ & $\mathrm{NpF}_{\mathrm{T}}$ & $\mathrm{RNE}(\%)$ \\
\hline D19 & 5.8 & 0.5 & 90.8 \\
D22 & 5.0 & 3.8 & 24.2 \\
D26 & 5.5 & 4.7 & 15.2 \\
\hline
\end{tabular}

Abbreviations: NpF, nymphs per incubated female mite; c, control group; ${ }_{\mathrm{T}}$, treatment group; RNE, reduction of nymph emergence treatment that also disrupts flea reproduction [17, 20]. Fluralaner's proven acaricidal effects after oral treatment suggested that a similar approach would be effective for the treatment of $D$. gallinae. The recent approval of a product containing fluralaner for the treatment of poultry red mite by the European Medicine Agency confirms this conclusion (Exzolt ${ }^{\mathrm{Tm}}$ authorization number: EU/2/17/212) [23, 28]. A $1 \%$ solution of fluralaner was orally administered to hens at the intended recommended dose regimen of 2 administrations of $0.5 \mathrm{mg}$ fluralaner $/ \mathrm{kg}$ body weight 7 days apart $[29,30]$. Instead of offering the medication to birds via free access to drinking water, hens were administered by gavage to ensure that each hen received not more than the minimum intended dose for the efficacy assessments. The systemic fluralaner dose regimen was well tolerated by hens.

\section{Acaricidal efficacy and effects on mite reproduction}

The study results confirm that oral administration of fluralaner to laying hens provides $99.3-100 \%$ acaricidal efficacy against $D$. gallinae infestation over a period of 15 days. Efficacy during this time frame was achieved within $12 \mathrm{~h}$ post-infestation preventing mites from laying any eggs. When efficacy assessments were conducted at $4 \mathrm{~h}$ post-infestation even this short period was sufficient to achieve high efficacy of $98.7-100 \%$ for a period of 12 days. The fast speed of kill (within 4-12 h) strongly contributes to the disruption of oviposition by poultry red mites, which generally deposit their eggs around $12 \mathrm{~h}$ after blood-feeding [1].

At 19 days after the initial administration to hens, the observed acaricidal efficacy of $74.8 \%$ did not reach common regulatory requirements [31, 32]. In the case that female mites managed to produce eggs, no ovicidal or larvicidal effects were observed but oviposition was considerably reduced by $50 \%$, and the emergence of protonymphs was substantially reduced by $90.8 \%$ (day 19). This demonstrates that the intended dose regimen of fluralaner for the treatment of poultry red mites in laying hens prevents mite reproduction even after the initial acaricidal effect diminishes.

Under optimal environmental conditions for mites, which are common in commercial poultry houses, a period of 2 weeks allows $D$. gallinae to complete its lifecycle twice and therefore, mites are able to build up large mite populations within a short time [33]. The presented data indicate that 2 oral administrations of fluralaner, given to hens 7 days apart, has the potential to achieve a sustainable elimination of $D$. gallinae from poultry houses, by providing a potent acaricidal effect for at least 2 weeks that is achieved within 4-12 h, and disrupts the mite's life-cycle. 


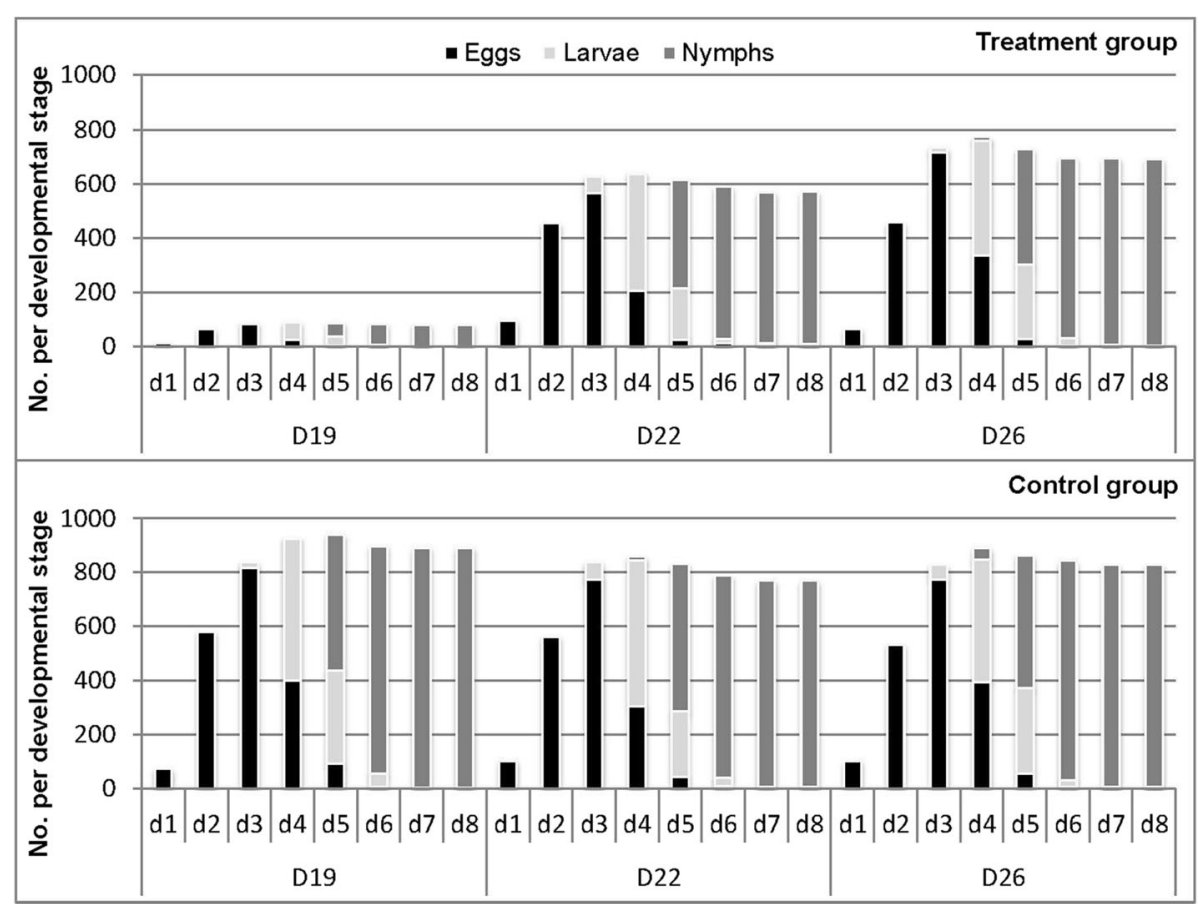

Fig. 2 Composition of mite populations in treated vs control group. Infestation days: 19 (D19), 22 (D22) and 26 (D26). Incubation and assessment days: days 1 to 8 (d1-d8)

\section{Conclusions}

Oral administration of a systemically acting acaricide provides an alternative treatment option in the control of D. gallinae in poultry. Two oral fluralaner administrations given to laying hens 7 days apart provide efficacy against the poultry red mite for at least 2 weeks. The rapid mite-killing effect is achieved within $4-8 \mathrm{~h}$ after mite feeding, preventing mite oviposition and disrupting the mite's life-cycle. Thus, oral administration of fluralaner can effectively treat existing poultry red mite infestation leading to the elimination of mites in poultry houses.

\section{Abbreviations}

ELF: Eggs per live female; EN: Newly observed eggs; LFM: Sum of live females; LH: Larval hatch; MM: Mite mortality; MR: Mortality rate; NCR: Nymphal conversion ratio; NpF: Nymphs per incubated female; RELF: Reduction of eggs per live female; RLH: Reduction of larval hatchability; RNC: Reduction of nymphal conversion; RNE: Reduction of nymph emergence; T: Tube

\section{Acknowledgements}

The authors would like to express their sincere appreciation to Mirjam Békefi Juergen Essig, Britta von Oepen, Volker Stippich, and Annette Schmitt for their assistance and contribution to this study.

\section{Funding}

Not applicable.

\section{Authors' contributions}

$M B, H Z, A H$ and $H W$ prepared the study design and protocol. MB and $H Z$ assisted in conducting the study and supervised the data analysis. EZ provided the statistical analysis. $\mathrm{MB}, \mathrm{HZ}$ and $\mathrm{HW}$ drafted the manuscript. All authors read and approved the final manuscript.

\section{Ethics approval}

The study was performed in compliance with German animal welfare regulations (Tierschutzgesetz) in a GLP-compliant facility and ethical approval was obtained prior to the beginning of the study by the responsible authorities (Landesuntersuchungsamt Rheinland-Pfalz). The study was designed as a randomized and negative controlled efficacy study.

\section{Consent of publication}

Not applicable.

\section{Competing interests}

The authors are employees of MSD Animal Health Innovation GmbH, Germany. This study was conducted as part of a research program to evaluate the safety and efficacy of fluralaner for the treatment of poultry red mites in hens.

\section{Publisher's Note}

Springer Nature remains neutral with regard to jurisdictional claims in published maps and institutional affiliations.

Received: 28 March 2017 Accepted: 13 November 2017

Published online: 02 December 2017

\section{References}

1. Chauve C. The poultry red mite Dermanyssus gallinae (de Geer, 1778): current situation and future prospects for control. Vet Parasitol. 1998;79:239-45.

2. Sparagano O, Pavlicevic A, Murano T, Camarda A, Sahibi H, Kilpinen O, et al. Prevalence and key figures for the poultry red mite Dermanyssus gallinae infections in poultry farm systems. Exp Appl Acarol. 2009:48:3-10.

3. Mul M. The poultry red mite, Dermanyssus gallinae (de Geer, 1778) - a small pest that packs a big punch. 2013. https://www.researchgate.net/ 
publication/258553789_Fact_sheet_Poultry_Red_Mite_in_Europe. Accessed 17 Feb 2017.

4. Huber K, Zenner L, Bicout DJ. Modelling population dynamics and response to management options in the poultry red mite Dermanyssus gallinae (Acari: Dermanyssidae). Vet Parasitol. 2011;176:65-73.

5. Valiente Moro C, De Luna CJ, Tod A, Guy JH, Sparagano OA, Zenner L. The poultry red mite (Dermanyssus gallinae): a potential vector of pathogenic agents. Exp Appl Acarol. 2009;48:93-104.

6. Valiente Moro C, Chauve C, Zenner L. Vectorial role of some dermanyssoid mites (Acari, Mesostigmata, Dermanyssoidea). Parasite. 2005;12:99-109.

7. Van Emous R. Verwachtte schade bloedluis 21 miljoen euro. Pluimveewebnl. 2017. https://www.pluimveeweb.nl/artikelen/2017/01/schade-bloedluis-21miljoen-euro/. Accessed 17 Feb 2017.

8. Valiente Moro C, Chauve C, Zenner L. Experimental infection of Salmonella enteritidis by the poultry red mite, Dermanyssus gallinae. Vet Parasitol. 2007; 146:329-36

9. Sigognault Flochlay A, Thomas E, Sparagano O. Poultry red mite (Dermanyssus gallinae) infestation: a broad impact parasitological disease that still remains a significant challenge for the egg-laying industry in Europe. Parasit Vectors. 2017;10:357.

10. George DR, Finn RD, Graham KM, Mul MF, Maurer V, Moro CV, Sparagano OA. Should the poultry red mite Dermanyssus gallinae be of wider concern for veterinary and medical science? Parasit Vectors. 2015;8:178.

11. Pritchard J, Kuster T, Sparagano O, Tomley F. Understanding the biology and control of the poultry red mite Dermanyssus gallinae: a review. Avian Pathol. 2015;44:143-53.

12. Kilpinen O, Roepstorff A, Permin A, Nørgaard-Nielsen G, Lawson LG, Simonsen HB. Influence of Dermanyssus gallinae and Ascaridia galli infections on behaviour and health of laying hens (Gallus gallus domesticus). Br Poult Sci. 2005:46:26-34

13. Abbas RZ, Colwell DD, labal Z, Khan A. Acaricidal drug resistance in poultry red mite (Dermanyssus gallinae) and approaches to its management. Worlds Poult Sci J. 2014;70:113-24.

14. Brannstrom S, Morrison DA, Mattsson JG, Chirico J. Genetic differences in internal transcribed spacer 1 between Dermanyssus gallinae from wild birds and domestic chickens. Med Vet Entomol. 2008;22:152-5.

15. Gassel M, Wolf C, Noack S, Williams H, llg T. The novel isoxazoline ectoparasiticide fluralaner: selective inhibition of arthropod gamma-aminobutyric acid- and Lglutamate-gated chloride channels and insecticidal/acaricidal activity. Insect Biochem Mol Biol. 2014;45:111-24.

16. Wengenmayer $\mathrm{C}$, Williams $\mathrm{H}$, Zschiesche E, Moritz A, Langenstein J, Roepke RK, Heckeroth AR. The speed of kill of fluralaner (Bravecto) against Ixodes ricinus ticks on dogs. Parasit Vectors. 2014;7:525.

17. Williams H, Young DR, Qureshi T, Zoller H, Heckeroth AR. Fluralaner, a novel isoxazoline, prevents flea (Ctenocephalides felis) reproduction in vitro and in a simulated home environment. Parasit Vectors. 2014;7:275.

18. Ozoe Y, Asahi M, Ozoe F, Nakahira K, Mita T. The antiparasitic isoxazoline A1443 is a potent blocker of insect ligand-gated chloride channels. Biochem Biophys Res Commun. 2010;391:744-9.

19. Bravecto EPAR. Summary for the public. European Medicines Agency. http:// www.ema.europa.eu/docs/en_GB/document_library/EPAR___Summary_for_ the_public/veterinary/002526/WC500163861.pdf. Accessed 17 Feb 2017.

20. Taenzler J, Wengenmayer C, Williams H, Fourie J, Zschiesche E, Roepke RK, Heckeroth AR. Onset of activity of fluralaner (BRAVECTO) against Ctenocephalides felis on dogs. Parasit Vectors. 2014;7:567.

21. Dryden MW, Smith V, Bennett T, Math L, Kallman J, Heaney K, Sun F. Efficacy of fluralaner flavored chews (Bravecto) administered to dogs against the adult cat flea, Ctenocephalides felis felis and egg production. Parasit Vectors. 2015;8:364.

22. Prohaczik A, Menge M, Huyghe B, Flochlay-Sigognault A, Traon GL. Safety of fluralaner oral solution, a novel systemic antiparasitic treatment for chickens, in laying hens after oral administration via drinking water. Parasit Vectors. 2017;10:363.

23. Exzolt ${ }^{\text {TM }}$ - Summary of opinion (initial authorization). European Medicines Agency. http://www.ema.europa.eu/docs/en_GB/document_library/ Summary_of_opinion_-_Initial_authorisation/veterinary/004344/ WC500229523.pdf. Accessed 13 Sept 2017

24. Packungsbeilage ByeMite $500 \mathrm{mg} / \mathrm{ml}$ Konzentrat zur Herstellung einer Sprühemulsion für Legehennen [Leaflet in German]. 2014

25. Schulz J, Berk J, Suhl J, Schrader L, Kaufhold S, Mewis I, et al. Characterization, mode of action, and efficacy of twelve silica-based acaricides against poultry red mite (Dermanyssus gallinae) in vitro. Parasitol Res. 2014;113:3167-75.
26. Meyer-Kuehling B, Pfister K, Mueller-Lindloff J, Heine J. Field efficacy of phoxim 50\% (ByeMite) against the poultry red mite Dermanyssus gallinae in battery cages stocked with laying hens. Vet Parasitol. 2007;147:289-96.

27. Nordenfors H, Höglund J, Tauson R, Chirico J. Effect of permethrin impregnated plastic strips on Dermanyssus gallinae in loose-housing systems for laying hens. Vet Parasitol. 2001;102:121-31.

28. European Commission. Exzolt ${ }^{\text {TM }}$ product information. Community register of veterinary medicinal products. http://ec.europa.eu/health/documents/ community-register/html/v212.htm. Accessed 15 Sept 2017.

29. Heckeroth AR, Zoller H, Flochlay-Sigognault A, Huyghe B. Use of isoxazoline derivatives for the treatment or prevention of arthropod infestations in poultry. Patent WO. 2015;2015091900:A1.

30. EMA. European public MRL assessment report (EPMAR), Fluralaner (poultry). 2017. http:/www.ema.europa.eu/docs/en_GB/document_library/Maximum_Residue_ Limits - Report/2017/02/WC500221753.pdf. Accessed 17 Feb 2017.

31. EMA. Guideline for the testing and evaluation of the efficacy of antiparasitic substances for the treatment and prevention of tick and flea infestation in dogs and cats. 2016. http://www.ema.europa.eu/docs/en_GB/document_library/ Scientific_guideline/2016/07/WC500210927.pdf. Accessed 17 Feb 2017.

32. Guideline: Demonstration of Efficacy of Ectoparasiticides. European Medicines Agency. 1994. http://www.ema.europa.eu/docs/en_GB/document_library/ Scientific_guideline/2009/10/WC500004662.pdf. Accessed 17 Feb 2017.

33. Kirkwood A. Longevity of the mites Dermanyssus gallinae and Liponyssus sylviarum. Exp Parasitol. 1963;14:358-66.

\section{Submit your next manuscript to BioMed Central and we will help you at every step:}

- We accept pre-submission inquiries

- Our selector tool helps you to find the most relevant journal

- We provide round the clock customer support

- Convenient online submission

- Thorough peer review

- Inclusion in PubMed and all major indexing services

- Maximum visibility for your research

Submit your manuscript at www.biomedcentral.com/submit
) Biomed Central 